\title{
EAl Endorsed Transactions

\section{DCE-CT Perfusion Parametric Coloured Image Using Steepest Gradient Approach Based on Time Frame}

\author{
Habib E. Ashoor* \\ Department of physics, College of science, University of Bahrain, Kingdom of Bahrain, P.O. Box:32038.
}

\begin{abstract}
Technological advances in medical imaging modality, counting higher special and temporal resolution, have been utilized to aid radiologist in diagnosing, prognostic and, hence, management of brain tumour. After tracer injection, CT scanning along with pharmacokinetic models are applied to measure several hemodynamic parameters used to describe microvasculature within a targeted region of interest (ROI). These parameters are a choice for diagnostic and prognostic and the likelihood of preferable treatment for brain tumour. The perfusion pixel-wise perfusion map image is a fingerprint of abnormality of ROI within brains that aid radiologist to separate between necrotic and neoplastic of tumour territory and to avoid arterial partial-volume averaging. This work aims to generate pixel-wise perfusion map colour image of brain by using the steepest gradient approach based on the time frame of tracer passage.
\end{abstract}

Keywords: perfusion image, DCE-CT, ROI, time frame, steepest gradient approach.

Received on 06 May 2020, accepted on 21 May 2020, published on 29 May 2020

Copyright (C) 2020 Habib E. Ashoor et al., licensed to EAI. This is an open access article distributed under the terms of the Creative Commons Attribution license, which permits unlimited use, distribution and reproduction in any medium so long as the original work is properly cited.

doi: 10.4108/eai.13-7-2018.164828

*Corresponding author. Email: hashoor@uob.edu.bh

\section{Introduction}

Angiogenesis is the procedure of proliferation of tumour cells, at which new vessels are formed to feed nutrients and oxygen for tumour growth where blood-brain barrier (BBB) is disrupted. On the other hand, angiogenesis plays an important role in forming and regulating the microvasculature and hence greatly influences tumour treatment [22] [3] [18] [4] [23].

Computed Tomography Dynamic Contrast-Enhanced (CT-DCE) technique has been used to measure certain parameters that describe the microvasculature [11] [14] [6]. Among these parameters is the blood perfusion which is measured by using the gradient approach.

Follow the injection of the tracer, normally iodinated contract agent, cine images of the area of interesting is generated to monitor the behaviour of the tracer first pass. By utilizing these cine images, hence, maps of perfusion parameters are generated, interpreted, and thereby aid in deciding whether a patient needs immediate thrombolytic treatment. This interpretation is critical in tailoring

treatment to each patient, and thus saving lives and reducing the possibility of severe disability.

The parametric maps are distant with regards to a certain diagnostic accuracy and further refinement of the techniques and methods in use are desired [24] [17] [19].

Many well-established tracer kinetic models are available that can provide useful estimates of microvasculature functional parameters. These models can be roughly classified into two streams [15]: the compartmental models and distributed parameter models developed by Lawrence and Lee [20]. Unlike the compartmental models, the distributed parameters models define the contrast agent within the vascular space as a function of time and distance along the length of a capillary.

Applying these models requires specific knowledge including the vascular and compartments, the volumes of the compartments constituting the model and the tracer permeability through the semi-membrane compartment barriers. Complexity, of the above models, leads researchers to use simpler and more straightforward model, such as gradient model [11] [21] [2]. 
The gradient model states that the maximum slope of the parenchyma curve has to be reached before venous outflow starts. The most advantage of this model is that there is no need to use the gamma variate fitting to smooth the AIF data [10].

Further work was done by Ashoor [1] to include the time frame of the bolus passage. In this work, based on pixel-wise analysis, colour map of cerebral blood flow based on Ashoor approach is established.

\section{Theoretical Background}

The Fick's principle, which is based on the basic law of conservation of mass, describes the detection of the transit of tracer bolus through a region as:

$$
\frac{\partial m}{\partial t}=F C_{a}(t)-F C_{v}(t)-m_{t}
$$

Where $\mathrm{m}$ is the amount of the tracer, $\mathrm{F}$ is the flow, $\mathrm{Ca}(\mathrm{t})$ is the feeding artery concentration, $\mathrm{Cv}(\mathrm{t})$ is the vein concentration and $\mathrm{mt}$ is the amount of tracer within the tissue of interest.

Assuming that the complete system is at systemic tracer steady state, the above equation can be expressed as:

$$
\begin{aligned}
& \frac{\partial m}{\partial t}=0=F C_{a}(t)-F C_{v}(t)-m_{t} \\
& o r \\
& F=\frac{m_{t}}{C_{a}(t)-C_{v}(t)}
\end{aligned}
$$

The above equation can be modified to make its application similar to the microsphere assumption, i.e. no outflow of the tracer from the tissue of interest $(\mathrm{Cv}(\mathrm{t})=0)$. The equation can be simplified as:

$$
\begin{aligned}
& F=\frac{m_{t}}{C_{a}(t)} \\
& \text { or } \\
& F=\frac{C_{t}(t) V_{t}}{C_{a}(t)} ; m_{t}=C_{t}(t) V_{t}
\end{aligned}
$$

Where $\operatorname{Ct}(\mathrm{t})$ is the tracer concentration that occupied $\mathrm{Vt}$, volume of the tissue of interest.
Hence, the perfusion within the tissue of interest can be written as:

$$
\frac{F}{V_{t}}=\text { perfusion }=\frac{C_{t}(t)}{C_{a}(t)}
$$

By using the stimulus-response theorem in which the tissue concentration can be written as:

$$
C_{t}(t)=\int_{t_{0}}^{T_{c}} C_{a}(t) d t
$$

Tc is the mean transit time of the tissue of interest, equation (4) can be written as:

$$
\frac{F}{V_{t}}=\text { perfusion }=\frac{\int_{t_{0}}^{T_{c}} C_{a}(t) d t}{C_{a}(t)}
$$

The optimum value of the ratio of the right-hand side occurs at $\mathrm{t} \leq \mathrm{Tc}$ at which the steepest gradient must be determined before washout of the tracer takes place from the tissue of interest.

Since the steepest gradient is located at the maximum of the feeding artery curve, usually sagittal sinus, the equation can be expressed in a simple form as:

$$
\begin{aligned}
& \frac{F}{V_{t}}=\text { perfusion }=\frac{\max \text { slope } \int_{t_{0}}^{T_{c}} C_{a}(t) d t}{\text { peak } C_{a}(t)} \\
& \text { or } \\
& \frac{F}{V_{t}}=\text { perfusion }=\frac{\text { max slope } C_{t}(t)}{\text { peak } C_{a}(t)}
\end{aligned}
$$


The advantage of using the proposed method by Ashoor [2] is that the initial stage data of the tracer passage is enough to generate colour map image of cerebral blood flow (CBF). For instance, utilising the data from the starting of the enhancement of CT scanner signal (starting point of the tracer's wash in) to the peak of AIF (starting point of the tracer's washout), where the tracer is confined, almost totally, within the voxel of interest (Fig. $1)$.

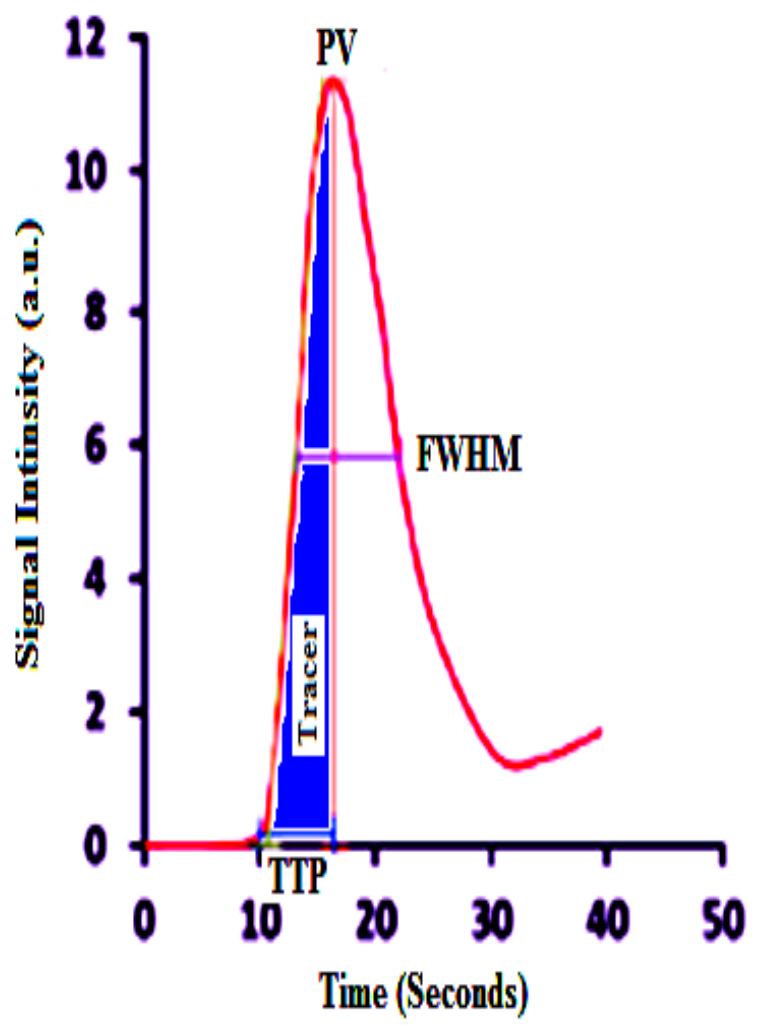

Fig.1. The voxel of interest

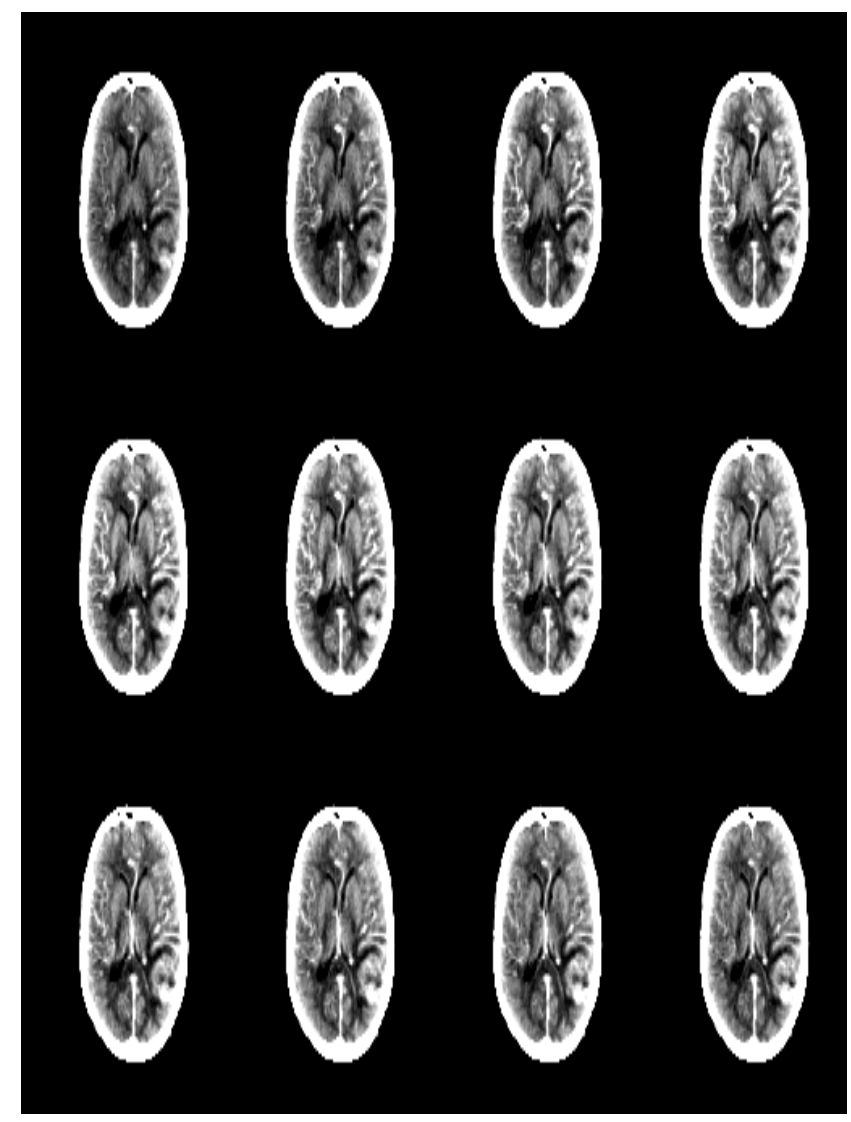

Fig.2. A sample of DCE-CT series images

Figure 1 showing a sample of DCE-CT series images acquired from the patient brain.

The perfusion map image is constructed by estimating the ratio between the maximum slope of the uptake curve and the peak of arterial input function [16] projecting individual pixel using equation (7).

\section{Results}

Based on the model developed in the previous section (steepest gradient method based on the time frame proposed by Ashoor [1], the perfusion image of blood flow is constructed (Fig.2). The active region of the tumour, as highlighted in the left image, represents the highest blood flow.

This area surrounds a dead area of less blood flow, the necrotic area. In the blood flow map image developed using the gradient method, the right one, indicates higher blood flow within the active area of the tumour (red). Clearly, it shows the enactive area as low blood flow (blue). 

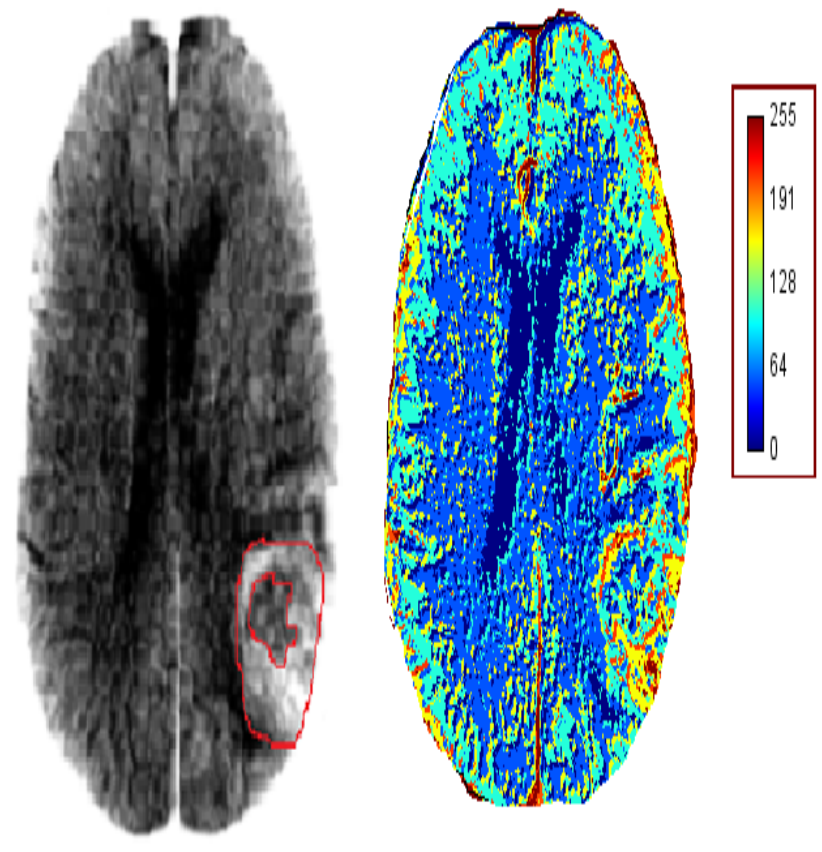

Fig.3. Compares the perfusion image HU

Figure 3 compares the perfusion image HU (right) generated using the TTP time frame steepest gradient approach based on the tracer passage time frame with $\mathrm{CT}$ grey image (left) HU.

\section{Discussion}

Nowadays dynamic enhanced contrast CT and computeraided decision CAD become valuable tools in the clinical environment aiding decision-makers (DM) in quantitative and diagnoses of abnormalities within the human brain. Consequently, this enhances favourable treatment, imageguided intervention, and surgical planning. Furthermore, the perfusion technique has been widely spread in the field of oncology as it provides valuable details about tumour grading and angiogenesis "in vivo [13]. Due to the heterogeneity of tumour cells, manual identification of the ROIs is not applicable since essential details about tumour morphology may be lost.

Hence, several attempts have been made to utilise the kinetic analysis of individual pixel data to generate a distribution map based on measured parameters that describe the microvasculature such as CBF [4] [12] [2] [7]. Measurement of CBF within the ROI, i.e. tumour, provides valuable details about the separation between necrotic area, low flow, and neoplastic area, high flow [5].

There exist several models used to establish blood flow coloured map image, however, the steepest gradient method is simple, least error and straight forward [11] [14] [9]. To generate the coloured map image of CBF by using the steepest gradient approach, tissue density curve
(TDC) and arterial input function (AIF) should be determined. AIF is the feeding artery of the tissue of interest that is programmatic to identify because of the complexity of the microvasculature [9]. Hence, to avoid arterial partial-volume averaging, superior sagittal sinus $[10]$ is used to establish AIF.

Furthermore, the steepest gradient approach based on the initial time frame of the tracer passage, proposed by Ashoor [2], is utilized to generate pixel-wise profusion coloured map image. After injection of iodinated tracer, intravenously, cine images (Figure 2) of targeted slices of brain are generated to monitor the behaviour of the tracer. Among the existing imaging modalities, $\mathrm{CT}$ is favourable, where the signal intensity is linearly proportional to the amount of the tracer within the ROI.

The main goal of image remapping and segmentation are to enhance images by dividing them into meaningful, homogeneous, and nonoverlapping or texture. The outcome of segmentation either an image of labels identifying each homogeneous region or a set of counters which describe the region boundaries. In this work, the map image is divided into colour to specify the more blood flow that can be identified by red colour (Figure 3). This assists decision-makers in the clinical environment to issue improved diagnoses, and hence, contributes overall treatments [8].

\section{Conclusion}

This study set out to assess the applicability of the steepest gradient based on the time frame by utilising CT dynamic imaging to generate the blood flow colour map. The most obvious finding to emerge from this study is that the steepest gradient based on time frame is practical less error approach allows to estimate the CBF. Furthermore, it is enough to build pixel-wise colour CBF map by using partial time course of tracer's passage that enabling to distinguish between necrotic area, low blood flow, and neoplastic area, high blood flow.

\section{Acknowledgements.}

This work has been done in the physics department at the University of Bahrain.

\section{References}

[1] Ashoor HE (2019). Optimal time frame required to accurately estimate Ktrans, vp and veusing graphical method: Simulation Study. 8th International Conference on Modeling Simulation and Applied Optimization (ICMSAO). Manama, Bahrain, IEEE.

[2] Bassani, B., D. Baci, et al. (2019). "Natural Killer Cells as Key Players of Tumor Progression and Angiogenesis: Old and Novel Tools to Divert Their Pro-Tumor Activities into Potent Anti-Tumor Effects." Cancers (Basel) 11(4).

[3] Blomley, M. J., A. McBride, et al. (1999). "Functional renal perfusion imaging with colour mapping: is it a useful 
adjunct to spiral $\mathrm{CT}$ of in the assessment of abdominal aortic aneurysm (AAA)?" Eur J Radiol 30(3): 214-20.

[4] Cenic, A., D. G. Nabavi, et al. (2000). "A CT Mehod to Measure Hemodynamic in Brain Tumors: Validation and Application of Cerebral Blood Flow Maps." Am J Neuroradiol 21: 462-470.

[5] Chen, X., J. Zou, et al. (2019). "Computed Tomography Perfusion Imaging Quality Affected by Different Input Arteries in Patients of Internal Carotid Artery Stenosis." Med Sci Monit 25: 9067-9072.

[6] De Vis, J. B., S. Song, et al. (2019). "Identifying perfusion deficits on CT perfusion images using temporal similarity perfusion (TSP) mapping." Eur Radiol 29(8): 4198-4206.

[7] Despotovic, I., Goossens, B, et al. (2014). "MRI Segmentation of the Human Brain: Challenges, Methods, and Applications." Computational and Mathematical Methods in Medicine 2015: 1-23.

[8] Kimura, T. and Kusahara, H. (2009). "Reference-based Maximum Upslope: A CBF Quantification Method without Using Arterial Input Function in Dynamic Suscetibility Contrast MRI." Magn Reson Med 8(3): 107120.

[9] Konig, M. (2003). "Brain perfusion CT in acute stroke: current status." European Journal of Radiology 45: S11S22.

[10] Miles, K. A. (1991). "Measurement of tissue perfusion by dynamic computed tomography." Br J Radiol 64(761): 409-12.

[11] Miles, K. A. (2003). "Staging of non-small-cell lung cancer with integrated PET and CT." N Engl J Med 349(12): 1188-90; author reply 1188-90.

[12] Miles, K. A. and Griffiths, M. R. (2003). "Perfusion CT: a worthwhile enhancement?" Br J Radiol 76(904): 220-31.

[13] Miles, K. A., M. Hayball, et al. (1991). "Colour perfusion imaging: a new application of computed tomography." Lancet 337(8742): 643-5.

[14] Murase, K. and S. Miyazaki (2007). "Error analysis of tumor blood flow measurement using dynamic contrastenhanced data and model-independent deconvolution analysis." Phys Med Biol 52(10): 2791-805.

[15] Nitzl, D., S. Ohlerth, et al. (2009). "Dynamic computed tomography to measure tissue perfusion in spontaneous canine tumors." Vet Radiol Ultrasound 50(4): 347-52.

[16] Oosterbroek, J., E. Bennink, et al. (2016). "Comparison of DCE-CT models for quantitative evaluation of $\mathrm{K}$ (trans) in larynx tumors." Phys Med Biol 60(9): 3759-73.

[17] Pedrosa, A. R., N. Bodrug, et al. (2019). "Tumor angiogenesis is differentially regulated by phosphorylation of endothelial cell focal adhesion kinase tyrosines-397 and -861." Cancer Res.

[18] Simonis, F. F., A. Sbrizzi, et al. (2016). "Improving the arterial input function in dynamic contrast enhanced MRI by fitting the signal in the complex plane." Magn Reson Med 76(4): 1236-45.

[19] St Lawrence, K. S. and T. Y. Lee (1998). "An adiabatic approximation to the tissue homogeneity model for water exchange in the brain: II. Experimental validation." J Cereb Blood Flow Metab 18(12): 1378-85.

[20] Vallee, J. P., F. Lazeyras, et al. (2000). "Absolute renal blood flow quantification by dynamic MRI and GdDTPA." Eur Radiol 10(8): 1245-52.

[21] van Elmpt, W., C. M. L. Zegers, et al. (2016). "Multiparametric imaging of patient and tumour heterogeneity in non-small-cell lung cancer: quantification of tumour hypoxia, metabolism and perfusion." Eur J Nucl Med Mol Imaging 43(2): 240-248.
[22] Yamada, A., K. Oyama, et al. (2019). "Dynamic contrastenhanced computed tomography diagnosis of primary liver cancers using transfer learning of pretrained convolutional neural networks: Is registration of multiphasic images necessary?" Int J Comput Assist Radiol Surg 14(8): 12951301.

[23] YW Lui, ER Tang, et al. (2010). "Evaluating of ct perfusion in the setting of cerebral ischemia: pattern and pitfalls." American jornal of radiology 31(9): 1552-1563. 